\title{
Dynamic Reliability Analysis of Gear Transmission System of Wind Turbine in Consideration of Randomness of Loadings and Parameters
}

\author{
Lei Wang, ${ }^{1}$ Tao Shen, ${ }^{1}$ Chen Chen, ${ }^{1}$ and Huitao Chen ${ }^{1,2}$ \\ ${ }^{1}$ School of Automation, Chongqing University, Chongqing 400044, China \\ ${ }^{2}$ School of Mechanical and Power Engineering, Henan Polytechnic University, Jiaozuo, Henan 454150, China \\ Correspondence should be addressed to Lei Wang; leiwang08@cqu.edu.cn
}

Received 30 December 2013; Accepted 19 January 2014; Published 6 March 2014

Academic Editor: Weichao Sun

Copyright (C) 2014 Lei Wang et al. This is an open access article distributed under the Creative Commons Attribution License, which permits unrestricted use, distribution, and reproduction in any medium, provided the original work is properly cited.

A dynamic model of gear transmission system of wind turbine is built with consideration of randomness of loads and parameters. The dynamic response of the system is obtained using the theory of random sampling and the Runge-Kutta method. According to rain flow counting principle, the dynamic meshing forces are converted into a series of luffing fatigue load spectra. The amplitude and frequency of the equivalent stress are obtained using equivalent method of Geber quadratic curve. Moreover, the dynamic reliability model of components and system is built according to the theory of probability of cumulative fatigue damage. The system reliability with the random variation of parameters is calculated and the influence of random parameters on dynamic reliability of components is analyzed. In the end, the results of the proposed method are compared with that of Monte Carlo method. This paper can be instrumental in the design of wind turbine gear transmission system with more advantageous dynamic reliability.

\section{Introduction}

Wind turbine generators usually work in a severe environment and suffer from the impact of random wind with varying directions and varying loads, as well as the strong gust, year after year. As a vital part of the transmission system of a wind turbine generator, the gear transmission system needs to withstand random dynamic loads and much higher fatigue cycles than any other transmission systems, thus making it possess the highest failure rate [1]. However, results of the general design and evaluation method of the gear transmission system, in which the random wind load is processed roughly as static load using statistic method, are not satisfied in solving the high failure rate problem of gear transmission system, which is a fundamental fact to restrict the life span of the whole wind turbine generator.

Many scholars worldwide have done many deep researches on the random vibration and dynamic reliability of random construction caused by random excitations [2-7]. However, their researches are relatively simple in choosing research objects, which can hardly conduct the design of dynamic reliability of gear transmission system. Recently, the dynamic issue of gear transmission system of wind turbine generator attracts more and more attention.

Peeters [8] and his fellows built the flexible multibody dynamics model of a wind turbine transmission system by applying multibody dynamics software and studied the natural frequency and vibration mode of the system. Caichao et al. [9] built the nonlinear dynamics model of wind turbine gearbox and analyzed the dynamic characteristic. Qin et al. $[10,11]$ studied the dynamic characteristic of the wind turbine transmission system with the dynamic torque input caused by simulated natural wind data. However, these studies did not consider the randomness of external loads, the uncertainty of gear transmission system material, and the geometric parameters. Nor did they analyze the dynamic reliability of the system. In actual wind farm, due to the fierce work environment and the uncertainty during the processing and assembly of the gears, the external excitations and the parameters of the gear transmission system are all random. 
So it is of great practical significance to develop a method to analyze the dynamic characteristics and the reliability under random wind conditions.

In this paper, we studied the gear transmission system of a 1.5 MW wind turbine. Elastic modulus, mass density, working tooth width, pitch circle diameter, and comprehensive transmission error were taken as random variables. The dynamic meshing force of gears was obtained by using the theory of random sampling and the Runge-Kutta method, taking into consideration the influence of external random load. On this basis, statistical processing of dynamic meshing force was done using rain flow counting principle and equivalent method of Geber quadratic curve. Dynamic reliability of components and the whole system were calculated according to the theory of probability of cumulative fatigue damage. In the end, the variation of the system dynamic reliability over time under variable parameters was studied. The effect of this variation to the system dynamic reliability was analyzed, and the results were compared with those of Monte Carlo method.

\section{Dynamic Model of Gear Transmission System}

2.1. Dynamic Model of Gear Transmission System. This paper studies the gear transmission system of a $1.5 \mathrm{MW}$ wind turbine generator, which contains one level of NGW planetary gear and two levels of parallel shaft gear. The structure diagram is shown in Figure 1.

Torsional vibration model of gear transmission system is built using centralized parameter method, as is shown in Figure 2. Variation of meshing stiffness, comprehensive transmission error, and other factors are taken into consideration in this model. The planetary gears are assumed to be uniformly distributed and have the same physical and geometrical parameters.

In Figure 2, $u_{c}, u_{s}, u_{p i}, u_{j}(i=1,2,3, j=1,2,3,4)$ represent the torsion displacement of planet carrier, sun gear, planetary gears, and medium and high speed level gears, respectively; $k_{s p i}, k_{r p i}$ represent the meshing stiffness of sun gear and planetary gear $i$ and the meshing stiffness of annular gear and planetary gear $i$, respectively; $k_{s 1}$ represents the torsional stiffness of the connecting shaft between the sun gear and gear $1 ; k_{23}$ represents the torsional stiffness of the connecting shaft between gear 2 and gear $3 ; k_{12}, k_{34}$ represent the meshing stiffness of the medium speed gears and the high speed gears; $c_{s p i}, c_{r p i}$ represent the meshing damping of sun gear, annular gear, and planetary gear $i ; c_{s 1}$ represents the torsional damper of connecting shaft between the sun gear and gear $1 ; c_{23}$ represents the torsional damper of connecting shaft between gear 2 and gear $3 ; c_{12}, c_{34}$ represent the meshing damping of the medium speed level gears and the high speed level gears; $e_{s p i}, e_{r p i}$ represent the transmission error of sun gear, annular gear, and planetary gear $i ; e_{12}, e_{34}$ represent the comprehensive transmission error of the medium speed and high speed level gears.

In the gear transmission system, the meshing of the gear pair and the gear meshing force and meshing displacement are all happening in the direction of the meshing line. In

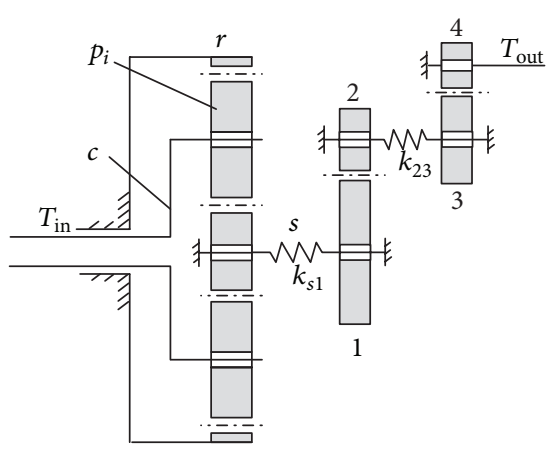

FIGURE 1: Schematic of gear transmission system of wind turbine. $p$ : planetary gear, $r$ : internal gear, $c$ : planet carrier, $s$ : sun gear, $1:$ small gear at medium-level speed, 2: large gear at medium-level speed, 3: small gear at high-level speed, 4: large gear at high-level speed, $T_{\text {in }}$ : input torque, and $T_{\text {out }}$ : output torque.

order to simplify the following analysis and calculation on the torsional vibration system, we replace the generalized coordinates in form of gear angular displacement with the ones in form of line displacement along the meshing line. Set the rotation angular displacement of each gear is $\theta_{i}$, respectively, $(i=c$, $p i, s, 1,2,3,4)$. According to the newly defined generalized coordinates, the rotation freedom of sun gear is converted to microdisplacement $u_{s}, u_{s}=r_{s} \theta_{s}\left(r_{s}\right.$ is the radius of the base circle of the sun gear) which is in the direction of the planetary gear meshing line. Similarly, the rotation freedom of planetary gear is converted to microdisplacement $u_{c}=r_{c} \theta_{c}$, also in the direction of meshing line, and so on.

The analysis of elastic deformation of each meshing force is as follows.

The elastic deformation in the direction of meshing force between the $i$ th planetary gear and the sun gear is

$$
\begin{aligned}
\delta_{s p i} & =r_{b c} \cos \alpha_{s p} \theta_{c}-r_{b p i} \theta_{p i}-r_{b s} \theta_{s}-e_{s p i} \\
& =u_{c} \cos \alpha_{s p}-u_{p i}-u_{s}-e_{s p i} .
\end{aligned}
$$

Its first derivative is

$$
\begin{aligned}
\dot{\delta}_{s p i} & =r_{b c} \cos \alpha_{s p} \dot{\theta}_{c}-r_{b p i} \dot{\theta}_{p i}-r_{b s} \dot{\theta}_{s}-\dot{e}_{s p i} \\
& =\dot{u}_{c} \cos \alpha_{s p}-\dot{u}_{p i}-\dot{u}_{s}-\dot{e}_{s p i} .
\end{aligned}
$$

The elastic deformation in the direction of meshing force between the $i$ th planetary gear and the internal ring gear is

$$
\begin{aligned}
\delta_{r p i} & =r_{b p i} \theta_{p i}-r_{b r} \theta_{r}+r_{b c} \cos \alpha_{r p} \theta_{c}-e_{r p i} \\
& =u_{p i}-u_{r}+u_{c} \cos \alpha_{r p}-e_{r p i} .
\end{aligned}
$$

Its first derivative is

$$
\begin{aligned}
\dot{\delta}_{r p i} & =r_{b p i} \dot{\theta}_{p i}-r_{b r} \dot{\theta}_{r}+r_{b c} \cos \alpha_{r p} \dot{\theta}_{c}-\dot{e}_{r p i} \\
& =\dot{u}_{p i}-\dot{u}_{r}+\dot{u}_{c} \cos \alpha_{r p}-\dot{e}_{r p i} .
\end{aligned}
$$

The elastic deformation in the direction of meshing force between the spur gear 1 and the spur gear 2 is

$$
\delta_{12}=r_{b 1} \theta_{1}+r_{b 2} \theta_{2}-e_{12}=u_{1}+u_{2}-e_{12} .
$$




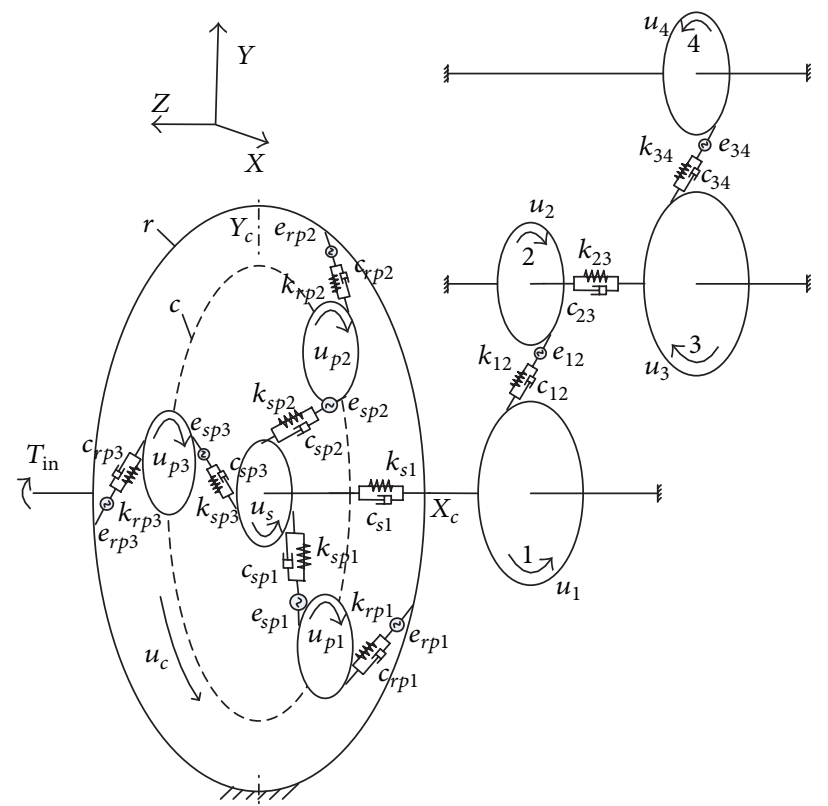

FIGURE 2: Torsional vibration model of gear transmission system.

Its first derivative is

$$
\dot{\delta}_{12}=r_{b 1} \dot{\theta}_{1}+r_{b 2} \dot{\theta}_{2}-\dot{e}_{12}=\dot{u}_{1}+\dot{u}_{2}-\dot{e}_{12} .
$$

The elastic deformation in the direction of meshing force between the spur gear 3 and the spur gear 4 is

$$
\delta_{34}=r_{b 3} \theta_{3}+r_{b 4} \theta_{4}-e_{34}=u_{3}+u_{4}-e_{34} .
$$

Its first derivative is

$$
\dot{\delta}_{34}=r_{b 3} \dot{\theta}_{3}+r_{b 4} \dot{\theta}_{4}-\dot{e}_{34}=\dot{u}_{3}+\dot{u}_{4}-\dot{e}_{34} .
$$

So in the gear transmission system, the relative displacements in the direction of meshing line of all gear pairs are

$$
\begin{gathered}
\delta_{s p i}=u_{c} \cos \alpha_{s p}-u_{p i}-u_{s}-e_{s p i}, \\
\delta_{r p i}=u_{p i}+u_{c} \cos \alpha_{r p}-e_{r p i}, \\
\delta_{n 12}=u_{1}+u_{2}-e_{12}, \\
\delta_{n 34}=u_{3}+u_{4}-e_{34} .
\end{gathered}
$$

Equation (10) is the vibration differential equations of the system based on Lagrange equation. Consider

$$
\begin{aligned}
& \left(\frac{I_{c}}{r_{b c}^{2}}\right) \ddot{u}_{c}+\sum_{i=1}^{3} c_{r p i} \cos \alpha_{r p i} \dot{\delta}_{r p i}+\sum_{i=1}^{3} c_{s p i} \cos \alpha_{s p i} \dot{\delta}_{s p i} \\
& +c_{u c} \dot{u}_{c}+\sum_{i=1}^{3} k_{r p i}(t) \cos \alpha_{r p i} \delta_{r p i} \\
& +\sum_{i=1}^{3} k_{s p i}(t) \cos \alpha_{s p i} \delta_{s p i}+k_{u c} u_{c}=\frac{T_{\mathrm{in}}}{r_{b c}},
\end{aligned}
$$

$$
\begin{aligned}
& \left(\frac{I_{p i}}{r_{b p i}^{2}}\right) \ddot{u}_{p i}-c_{s p i} \dot{\delta}_{s p i}+c_{r p i} \dot{\delta}_{r p i}-k_{s p i}(t) \delta_{s p i} \\
& +k_{r p i}(t) \delta_{r p i}=0, \\
& \left(\frac{I_{s}}{r_{b s}^{2}}\right) \ddot{u}_{s}-\sum_{i=1}^{3} c_{s p i} \dot{\delta}_{s p i}+c_{s 1}\left(\frac{\dot{u}_{s}}{r_{b s}^{2}}-\frac{\dot{u}_{1}}{r_{b s} r_{b 1}}\right) \\
& -\sum_{i=1}^{3} k_{s p i}(t) \delta_{s p i}+k_{s 1}\left(\frac{u_{s}}{r_{b s}^{2}}-\frac{u_{1}}{r_{b s} r_{b 1}}\right)=\frac{T_{s}}{r_{b s}}, \\
& \left(\frac{I_{1}}{r_{b 1}^{2}}\right) \ddot{u}_{1}+c_{12} \dot{\delta}_{n 12}+c_{s 1}\left(\frac{\dot{u}_{1}}{r_{b 1}^{2}}-\frac{\dot{u}_{s}}{r_{b s} r_{b 1}}\right) \\
& +k_{12}(t) \delta_{n 12}+k_{s 1}\left(\frac{u_{1}}{r_{b 1}^{2}}-\frac{u_{s}}{r_{b s} r_{b 1}}\right)=\frac{T_{1}}{r_{b 1}}, \\
& \left(\frac{I_{2}}{r_{b 2}^{2}}\right) \ddot{u}_{2}+c_{12} \dot{\delta}_{n 12}+c_{23}\left(\frac{\dot{u}_{2}}{r_{b 2}^{2}}-\frac{\dot{u}_{3}}{r_{b 2} r_{b 3}}\right) \\
& +k_{12}(t) \delta_{n 12}+k_{23}\left(\frac{u_{2}}{r_{b 2}^{2}}-\frac{u_{3}}{r_{b 2} r_{b 3}}\right)=\frac{T_{2}}{r_{b 2}}, \\
& \left(\frac{I_{3}}{r_{b 3}^{2}}\right) \ddot{u}_{3}+c_{34} \dot{\delta}_{n 34}+c_{23}\left(\frac{\dot{u}_{3}}{r_{b 3}^{2}}-\frac{\dot{u}_{2}}{r_{b 2} r_{b 3}}\right) \\
& +k_{34}(t) \delta_{n 34}+k_{23}\left(\frac{u_{3}}{r_{b 3}^{2}}-\frac{u_{2}}{r_{b 2} r_{b 3}}\right)=\frac{T_{3}}{r_{b 3}}, \\
& \left(\frac{I_{4}}{r_{b 4}^{2}}\right) \ddot{u}_{4}+c_{34} \dot{\delta}_{n 34}+k_{34}(t) \delta_{n 34}=-\frac{T_{\text {out }}}{r_{b 4}} \text {, }
\end{aligned}
$$

where $r_{b c}, r_{b s}, r_{b p i}, r_{b j}$ represent the base circle radii of planet carrier, sun gear, planetary gear, the medium speed gear, and the high speed gear, respectively. $T_{\text {in }}, T_{\text {out }}$ are the input torque and output torque of the system, respectively. 
Equation (10) can be simplified as matrix form:

$$
M \ddot{x}+C \dot{x}+K(t) x=T(t),
$$

where $x$ represents generalized displacement vector of the system. $x=\left[u_{c}, u_{p 1}, u_{p 2}, u_{p 3}, u_{s}, u_{1}, u_{2}, u_{3}\right]^{T} ; M, C, K(t)$ are 9 order matrixes of mass, damp, and time varying stiffnesses; $T(t)$ is the vector of external load caused by external input torque.

2.2. Solving of Equations. The common approaches to solve the equations of the dynamic model of gear transmission system are analytical method and numerical simulation method. The former includes piecewise linearization method and harmonic balance method, while the latter includes Newmark$\beta$ method and Runge-Kutta method. Unfortunately, their objects are all determined systems, thus making it impossible to solve the dynamic response of random system by directly applying these existing methods. In this paper, the random problem is converted into a determined one by sampling the random parameters in every moment.

The specific steps are as follows.

(1) Determine the elastic modulus, mass density, working tooth width, pitch circle diameter, and the distribution of comprehensive transmission error of the gear material.

(2) Divide the external excitation into $N$ portions equally, and determine each integration time step $\Delta t$ based on N.

(3) Assume that the rest of the parameters are determined when studying the influence of the response brought by variation of one single parameter. Sample the varying parameter at each sampling time.

(4) Obtain the dynamic response at one moment by calculating the dynamic equations with the sample results, using fixed step Runge-Kutta method.

(5) Sample the parameters of the next moment, and calculate the dynamic response at this moment.

(6) Change to another parameter, and repeat (2)-(5).

After getting the statistical characteristics of vibration displacement and vibration velocity of the system at each moment, the dynamic meshing force of each gear pair can be derived from the following equation:

$$
W_{i j}=k_{i j} \cdot\left(X_{i j}-x_{i j}-e_{i j}\right)+c_{i j}\left(\dot{X}_{i j}-\dot{x}_{i j}-\dot{e}_{i j}\right),
$$

in which $k_{i j}, c_{i j}$, and $X_{i j}$, respectively, are the meshing stiffness, damping coefficient, and relative displacement between gears $i$ and $j ; x_{i j}$ is the equivalent displacement of center displacement between the meshing lines of gears $i$ and $j$; $e_{i j}$ is the comprehensive meshing error of gears $i$ and $j$.

\section{Analysis of System Excitations}

3.1. External Excitation. The randomness of system load is mainly caused by external wind load. The variation of external excitation of the gear transmission system is determined by the random wind velocity. In this paper, stochastic volatility (SV) model is built to obtain the random wind velocity sequence in the wind farm. Then the external excitation of the transmission system is calculated according to the theory of aerodynamic.

SV model is a method of time series analysis, which is used in research on analyzing wind velocity. The main feature of SV model is to regard volatility as an implicit variable that cannot be observed. The basic form of SV model is [12]

$$
\begin{gathered}
v_{t}=\varepsilon_{t}+E\left(y_{t} \psi_{t-1}\right)=\sigma_{t} z_{t}, \\
\ln \left(\sigma_{t}^{2}\right)=a+\varphi \ln \left(\sigma_{t-1}^{2}\right)+\sigma_{\eta} \eta_{t},
\end{gathered}
$$

where $v_{t}$ is the amplitude of volatility; $\varepsilon_{t}$ is kurtosis; $E\left(y_{t}\right.$ । $\left.\psi_{t-1}\right)$ is the conditional mean of $v_{t}$ calculated from the information sampled at $t-1 ; \sigma_{t}$ is the conditional mean square deviation; $z_{t}$ follows a normal distribution with 0 mean and 1 variance; $a$ is a constant which reflects the average volatility; $\varphi$ is a parameter which reflects sustainability; $\sigma_{\eta}$ is the mean square deviation of volatility disturbance; $\eta_{t}, z_{t}$ follow independent normal distributions with 0 mean and 1 variance.

The random wind velocity simulated by SV model is taken as the input of the gear transmission system of wind turbine. Based on the aerodynamic theory, the input power of the transmission system is [10]

$$
p_{\text {in }}=\frac{1}{2} \rho S^{2} v_{t}^{3} C_{p}
$$

where $p_{\text {in }}$ is the input power of transmission system; $\rho$ is air density; $S$ is the sweeping area of wind turbine; $C_{p}$ is wind energy utilization factor; $v_{t}$ is the wind velocity simulated from SV model far from wind turbines.

The external excitation of the system is the torque ripple caused by random wind velocity. The torques from the input and output sides, respectively, are

$$
\begin{aligned}
& T_{\text {in }}=\frac{p_{\text {in }}}{\omega}, \\
& T_{\text {out }}=\frac{T_{\text {in }}}{i},
\end{aligned}
$$

where $\omega$ is the angular velocity of wind turbines; $i$ is the transmission ratio of the gear transmission system.

3.2. Stiffness Excitation. Stiffness excitation is a parametric excitation caused by the variation of meshing stiffness during the meshing process. Due to many influencing factors during machining and assembling, the size and material of the gear transmission components vary randomly, such as elastic modulus and working tooth width. In this paper, gear's stiffness is assumed to be a superposition of a sine wave and a random wave. The former is expressed by limited harmonic waves of Fourier series, and the latter is expressed by standard 
normal distribution function. Therefore, the comprehensive meshing stiffness of gears is as follows:

$$
k(t)=k_{m}+\sum_{j=1}^{m}\left[k_{j 1} \cos j \omega t+k_{j 2} \sin j \omega t\right]+\varepsilon_{1},
$$

where $k_{m}$ is the average meshing stiffness of the gear pairs; $k_{j 1}$ and $k_{j 2}$ are the meshing stiffness of harmonic waves; $\omega$ is meshing frequency; $\varepsilon_{1}$ is stiffness fluctuation caused by the variation of elastic modulus, which follows a normal distribution.

3.3. Error Excitation. Meshing error is a displacement excitation, which is related to the machining accuracy of the gears. The gear error and base pitch error can be expressed as a superposition of a sine wave and a random wave as follows [13]:

$$
e(t)=e_{m}+e_{r} \sin \left(\frac{2 \pi \omega t}{T}+\varphi\right)+\varepsilon_{2},
$$

where $e_{m}$ and $e_{r}$ are the offset and amplitude of the gear meshing error; $T, \omega, \varphi$ are the meshing period of the gear pair, meshing frequency, and initial phase angle; $\varepsilon_{2}$ is the fluctuation of comprehensive transmission error caused by machining and assembling, which is assumed to follow a normal distribution. In this paper, the gear accuracy is presumed to be grade 6 , and parameters involved are based on GB/T 10095-1988 standard.

\section{Analysis of System Dynamic Reliability}

4.1. Random Fatigue Load Spectrum of Gear Transmission System. Load-time history of each gear pair can be obtained by the dynamic gear transmission model built before. Then to analyze the fatigue reliability of the system, the loadtime history is converted into a series of complete cycles. The main converting methods are peak counting method, cycle counting method, rain flow counting method, and so forth.

In this paper, we count the dynamic meshing force of each gear pair circularly according to the rain flow counting principle [14-16] in order to obtain the frequency of luffing fatigue load. As is shown in Figure 5, the mean stress of the gear pairs follows a normal distribution approximately, and the amplitude of the stress follows Weibull distribution approximately.

In order to analyze the fatigue life of the transmission system, the equivalent amplitude and frequency of the system stress are obtained by using equivalent method of Geber quadratic curve. The Geber quadratic curve formula is [17]

$$
S_{\mathrm{eqv}}=S_{a} \frac{\sigma_{b}^{2}}{\sigma_{b}^{2}-S_{m}^{\prime 2}},
$$

where $S_{a}$ is the amplitude of stress after the conversion; $S_{m}^{\prime}$ is the mean stress of $S-N$ curve of the given material; $S_{\text {eqv }}$ is the equivalent stress corresponding to $S_{\text {eqv }}$ with equal lifetime.
4.2. Dynamic Reliability Model of Gear Transmission Components and System. Fatigue failure of the components is caused by the accumulation of material internal damage. As the number of stress cycles increases, the material internal damage exacerbates and the structural life decreases. Theory of probability fatigue damage is based on the fatigue damage evolution, which demonstrates the irreversibility and the randomness of fatigue damage. The main reason of the randomness of fatigue damage lies in the characteristics of the material, the geometric dimensions of the test pieces, and the uncertainty of external load.

The decay rate of the material ultimate stress generally follows distribution as [18-20]

$$
\frac{d \sigma_{u}}{d n}=\frac{-f\left(S_{\max }, f_{c}, r\right)}{c \sigma_{u}^{c}},
$$

where $S_{\max }$ is the maximin cyclic stress; $f_{c}$ is the cycle frequency; $r$ is cyclic stress ratio; $c$ is a constant.

The remaining ultimate stress of the component material after $n$ cycles is

$$
\sigma_{u}(n)=\sigma_{u 0}\left\{1-\left[1-\left(\frac{S_{\max }}{\sigma_{u 0}}\right)^{c}\right] \frac{n}{n_{i}}\right\}^{1 / c},
$$

where $\sigma_{u}(n)$ is the remaining ultimate stress after $n$ cycles; $\sigma_{u 0}$ is the ultimate stress when materials are in good condition; $n_{i}$ is the number of ultimate cycles.

The damage index of component under the level $i$ luffing cyclic stress after $n$ cycles is

$$
\Delta D=\sum_{i=1}^{n} \frac{\left(1-\left(\sigma_{u}\left(n_{i}\right) / \sigma_{u}\left(n_{i-1}\right)\right)^{c}\right)}{\left(1-\left(S_{\max i} / \sigma_{u}\left(n_{i-1}\right)\right)^{c}\right)},
$$

where $\sigma_{u}\left(n_{i}\right)$ and $\sigma_{u}\left(n_{i-1}\right)$ are the remaining ultimate stress under the level $i$ and level $i-1$ stress; $S_{\max i}$ is the maximum stress of level $i$ stress cycles.

Suppose $\sigma_{u}\left(n_{i}\right)(i=1,2, \ldots, n)$ are independent random variables from each other. $\sigma_{u}=\left(\sigma_{u 1}, \sigma_{u 2}, \ldots, \sigma_{u n}\right)$, which are means of $\sigma_{u}\left(n_{1}\right), \sigma_{u}\left(n_{2}\right), \ldots, \sigma_{u}\left(n_{n}\right)$ in $(6)$, respectively, are expanded into the Taylor series. Then the approximate mean $\mu_{\Delta D}$ and standard deviation $\sigma_{\Delta D}$ of the damage index $\Delta D$ are obtained by choosing the linear terms from the Taylor expansion. Consider

$$
\begin{aligned}
\mu_{\Delta D}= & g\left(\sigma_{D}\right)+\left.\sum_{i=1}^{n}\left(\sigma_{u}\left(n_{i}\right)-\sigma_{u i}\right) \frac{\partial g}{\partial \sigma_{u}\left(n_{i}\right)}\right|_{\sigma_{u i}} \\
& +\left.\frac{1}{2} \sum_{i=1}^{n}\left(\sigma_{u}\left(n_{i}\right)-\sigma_{u i}\right)^{2} \frac{\partial^{2} g}{\partial \sigma_{u}^{2}\left(n_{i}\right)}\right|_{\sigma_{u i}}+\cdots \\
\sigma_{\Delta D}= & \sqrt{E\left(\Delta D_{i}-\mu_{\Delta D}\right)^{2}}+ \\
= & \sqrt{\left.\sum_{i=1}^{n}\left(\sigma_{u}\left(n_{i}\right)-\sigma_{u i}\right)^{2} \frac{\partial^{2} g}{\partial \sigma_{u}^{2}\left(n_{i}\right)}\right|_{\sigma_{u i}}} .
\end{aligned}
$$


TABLE 1: Geometric parameters of gear transmission system of wind turbine.

\begin{tabular}{|c|c|c|c|c|c|c|}
\hline Low-level speed & $\begin{array}{c}\text { Number of sun gear } \\
\text { teeth } Z_{s} \\
27\end{array}$ & $\begin{array}{c}\text { Number of planetary } \\
\text { gear teeth } Z_{p} \\
44\end{array}$ & $\begin{array}{c}\text { Number of internal } \\
\text { gear teeth } Z_{r} \\
117\end{array}$ & $\begin{array}{c}\text { Number of } \\
\text { molds } m \\
13\end{array}$ & $\begin{array}{c}\text { Meshing angle } \\
\alpha_{s p}\left({ }^{\circ}\right) \\
23.0000\end{array}$ & $\begin{array}{c}\text { Meshing angle } \\
\alpha_{r p}\left({ }^{\circ}\right) \\
21.0000\end{array}$ \\
\hline $\begin{array}{l}\text { Medium-level } \\
\text { speed }\end{array}$ & \multicolumn{2}{|c|}{$\begin{array}{c}\text { Number of driving gears } Z_{1} \\
104\end{array}$} & \multicolumn{2}{|c|}{$\begin{array}{c}\text { Number of driven gears } Z_{2} \\
23\end{array}$} & \multicolumn{2}{|c|}{$\begin{array}{c}\text { Meshing angle } \alpha\left(^{\circ}\right) \\
21.0000\end{array}$} \\
\hline High-level speed & \multicolumn{2}{|c|}{$\begin{array}{c}\text { Number of driving gears } Z_{3} \\
98\end{array}$} & \multicolumn{2}{|c|}{$\begin{array}{c}\text { Number of driven gears } Z_{4} \\
25\end{array}$} & \multicolumn{2}{|c|}{$\begin{array}{l}\text { Meshing angle } \alpha\left(^{\circ}\right) \\
21.0000\end{array}$} \\
\hline
\end{tabular}

In engineering application, random index of fatigue cumulative damage is supposed to follow a lognormal distribution. That makes the distribution of $D$ be

$$
f_{D}(D)= \begin{cases}\frac{1}{\sqrt{2 \pi} \sigma_{D} D} \exp \left[-\frac{\left(\ln D-\mu_{D}\right)^{2}}{2 \sigma_{D}^{2}}\right], & D>0, \\ 0, & D \leq 0 .\end{cases}
$$

where $\mu_{D}$ is the logarithmic mean of cumulative damage $D$; $\sigma_{D}$ is the logarithmic mean square deviation of $D$.

Based on the theory of probability fatigue cumulative damage, the structural dynamic reliability of one moment is

$$
R=R\left(D<D_{0}\right)=\int_{0}^{D_{0}} f_{D}(y) d y
$$

where $D_{0}$ is the limit of damage index.

According to Figure 1, in the gear transmission system, the gears are connected in series; thus the whole system will fail if one of the gears fails. In other words, the system reliability is based on the reliability of each gear. Therefore, the reliability model gear transmission system is as follows:

$$
R(t)=R_{c}(t) \cdot \prod_{i=1}^{3} R_{p i}(t) \cdot R_{r}(t) \cdot R_{s}(t) \cdot \prod_{j=1}^{4} R_{j}(t),
$$

where $i$ is the number of planetary gears; $j$ is the number of medium speed level and high speed level gears.

4.3. Calculation of System Dynamic Reliability. The reliability of the gear transmission system is calculated using Matlab software. Based on the analysis before, the steps of the program are as follows.

(1) Take the random input torque of the gear transmission system as the extern excitation. Get the dynamic meshing force and its statistic characteristics by using the numerical integration method.

(2) Process the data of meshing force by rain flow counting method. Then calculate the equivalent stress amplitude and frequency by using equivalent method of Geber quadratic curve.

(3) Calculate structural fatigue damage under the luffing stress.

(4) Calculate cumulative fatigue damage of arbitrary time $t$ under several stress cycles.

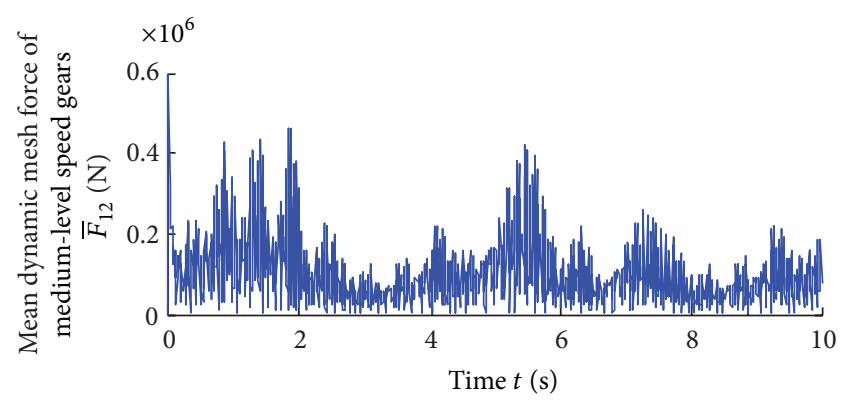

FIgURE 3: Mean dynamic meshing force of medium-level speed gears.

(5) Calculate the structural limit value of fatigue damage.

(6) By giving a random cumulative damage index, apply the equation of dynamic reliability to calculate the reliability of each gear when the tooth surface reaches the contact fatigue limit and the tooth root reaches the bending fatigue limit.

(7) Calculate the dynamic reliability of the gear transmission system using (25).

\section{Analysis of Examples}

The study object of the example research is the gear transmission system of a $1.5 \mathrm{MW}$ wind turbine. Here are some parameters used in this research: the rated power of the wind turbine is $1.5 \mathrm{MW}$; the impeller diameter is $70 \mathrm{~m}$; the designed impeller speed is $14.8 \mathrm{r} / \mathrm{min}$; average wind speed of the wind farm is $14.3 \mathrm{~m} / \mathrm{s}$; wind density is $1.21 \mathrm{~kg} / \mathrm{m}^{3}$; wind energy utilization factor is 0.32 ; system transmission ratio is 94.53 . Suppose the strength of the material and the coefficient of performance both follow a normal distribution, while other parameters are constant. Suppose the material of planetary gear is $40 \mathrm{Cr}$ and the material of medium-level speed and high-level speed gear is $20 \mathrm{CrMnTi}$. Other parameters of the system are shown in Table 1 .

By solving the dynamic equation (10) of the system, vibration displacement and vibration velocity of the gears at each moment are obtained, as well as their statistical characteristics. By solving (11), the meshing forces of each gear are obtained. Figure 3 shows the curve of mean dynamic meshing force of medium-level speed gears. Figure 4 shows 


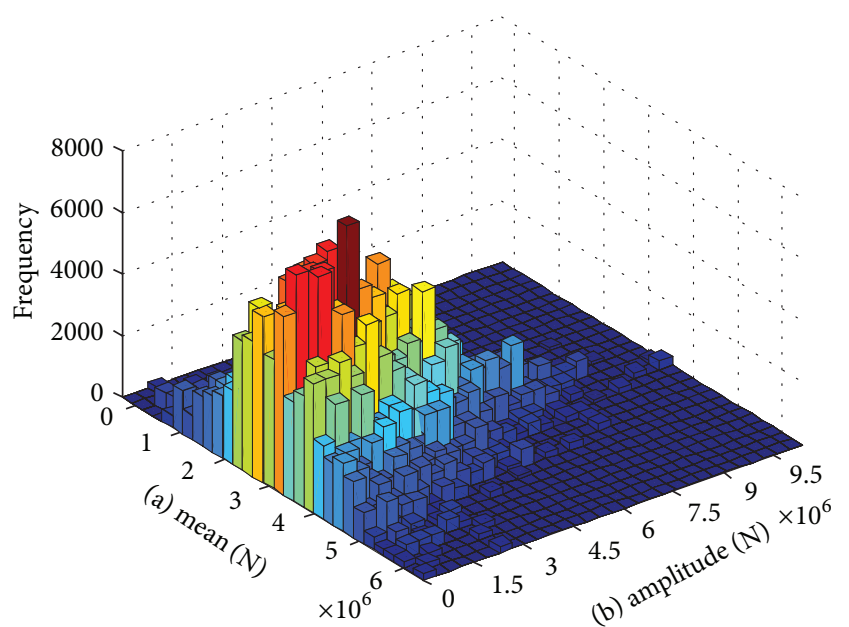

Figure 4: Luffing load spectrum of medium-level speed gears.

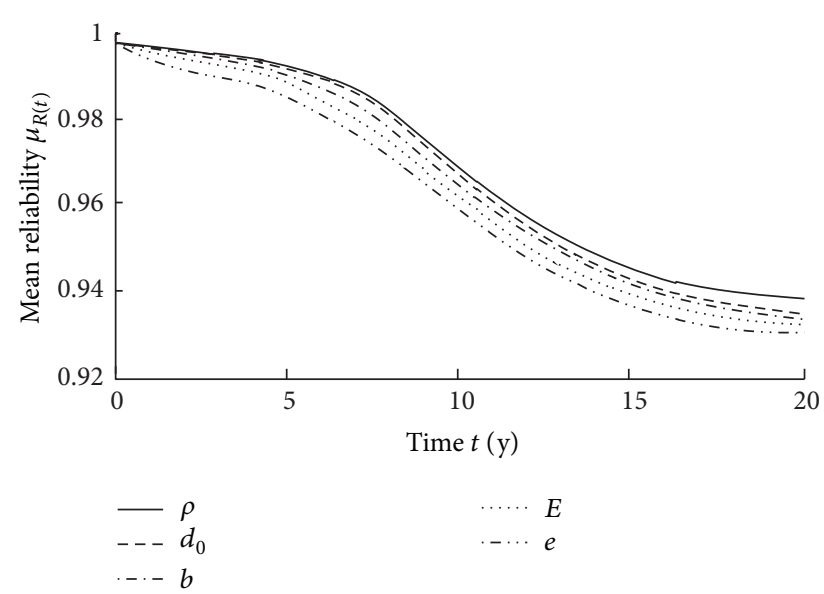

FIGURE 5: Dynamic reliability of system when variation coefficient of random parameter is 0 .

the luffing load spectrum of medium-level speed gears based on the theory of rain flow counting method.

We define the ratio of the mean square error and the mean of the system parameters as their variation coefficient. Figures 5-7 show the variation of system dynamic reliability over time with the variation coefficient being $0,0.1$, and 0.3 , respectively. As is demonstrated in Figure 5, the comprehensive transmission error $e$ has the greatest influence on the system reliability, followed by the elastic modulus of gear material $E$, contact tooth width $B$, and pitch circle diameter $d_{0}$. Mass density $\rho$ has the least influence. By comparing Figures 6 and 7, we can also learn that, with the variation of random parameters increases, the system gets more reliable.

Table 2 shows the dynamic reliability of each component in the transmission system as the comprehensive transmission error $e$ and mass density $\rho$ vary randomly, when $t=$ $6.3 \times 10^{8} \mathrm{~s}$. The table also shows that when the comprehensive transmission error $e$ and mass density $\rho$ are $0,0.1$, and 0.3 , respectively, in the whole transmission system, planetary gear system has the highest dynamic reliability, followed by

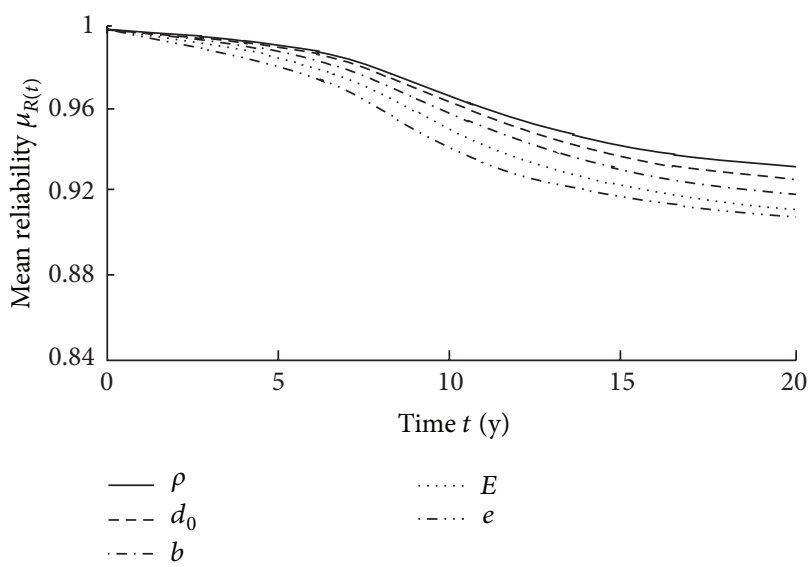

FIGURE 6: Dynamic reliability of system when variation coefficient of random parameter is 0.1 .

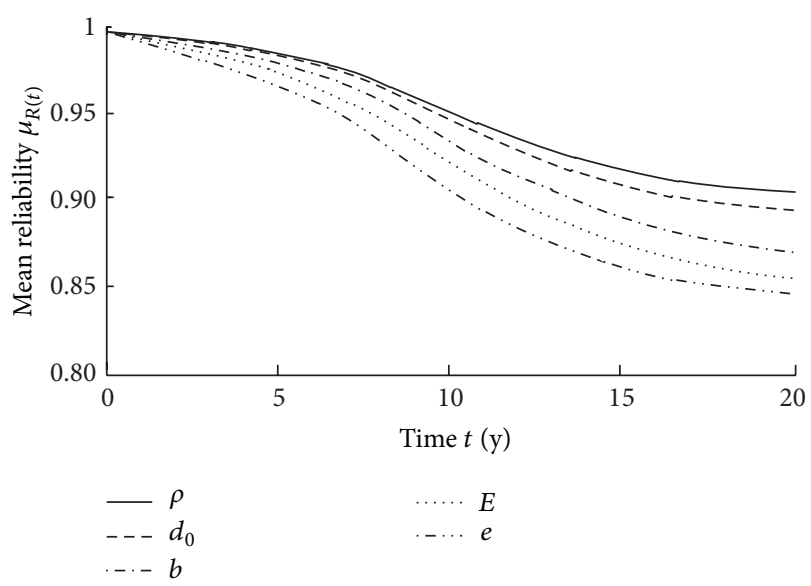

FIGURE 7: Dynamic reliability of system when variation coefficient of random parameter is 0.3 .

the medium speed level gears, while high speed level gear is the least reliable. In the planetary gear system, internal gears have the highest reliability, followed by the planetary gear, while the sun gear is the least reliable. In the medium and high speed level gears, large gears are more dynamicreliable than the small ones. The dynamic reliability of the gear transmission system reduces, and the dispersion degree of the system increases with the increase of the parameters' variation.

We obtained the statistical properties of the dynamic reliability of the high speed level gears through 20000 simulations when $t=6.3 \times 10^{8} \mathrm{~s}$, using Monte Carlo method, and compared the results with this paper, as is shown in Table 3. The method proposed in this paper is more accurate than Monte Carlo method.

\section{Conclusions}

In this paper, the dynamic reliability of the gear transmission system of a $1.5 \mathrm{MW}$ wind turbine, with consideration of 


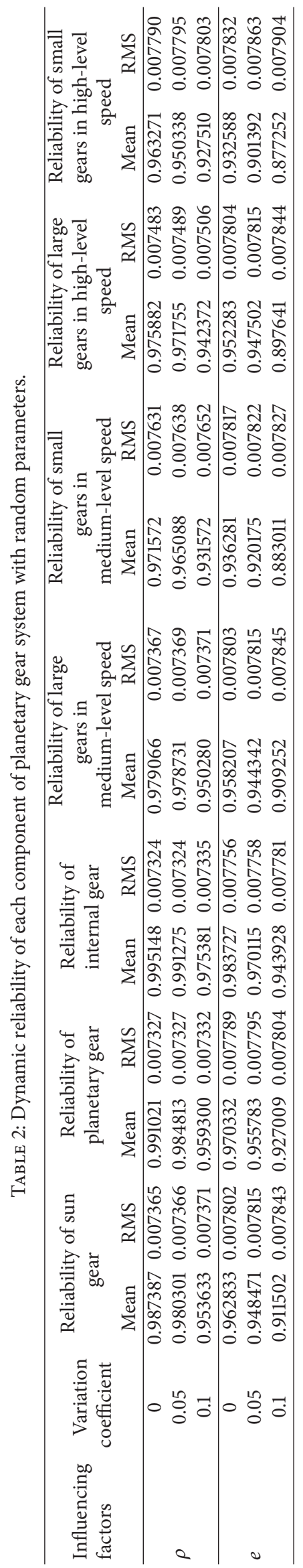


TABle 3: The comparison of dynamic reliability of big gear of high speed gear system.

\begin{tabular}{lccccc}
\hline \multirow{2}{*}{ Random parameters } & Variation coefficient & \multicolumn{2}{c}{ Proposed method } & \multicolumn{2}{c}{ Monte Carlo method } \\
& & Mean of $R(t)$ & Root mean square of $R(t)$ & Mean of $R(t)$ & Root mean square of $R(t)$ \\
\hline$B$ & 0.1 & 0.943257 & 0.008450 & 0.940832 & 0.008541 \\
$E$ & 0.1 & 0.940144 & 0.008671 & 0.939124 & 0.008454 \\
$\rho$ & 0.1 & 0.944946 & 0.008377 & 0.945271 & 0.008272 \\
$d_{0}$ & 0.1 & 0.946826 & 0.008498 & 0.939567 & 0.008157 \\
$e$ & 0.1 & 0.909033 & 0.008870 & 0.897354 & 0.008625 \\
\hline
\end{tabular}

randomness of load and system parameters, is analyzed by applying the theory of probability of cumulative fatigue damage. The main contributions and conclusions of this paper are the following.

(1) The dynamic model of the gear transmission of wind turbine is built. In consideration of the randomness of the load and gear parameters, the dynamic response of the system is obtained by utilizing the random sampling method and Runge-Kutta method. The statistical properties of the meshing force of components in the gear transmission system are obtained by statistic method.

(2) By applying the method of rain flow counting, the time history of the components meshing force is converted into a series of luffing load spectra, and the equivalent stress amplitude and frequency are calculated according to the equivalent method of Geber quadratic curve.

(3) The dynamic reliability model of the transmission system and gear components are built according to the principle of probability fatigue damage cumulative. Variation of the system reliability over time is calculated when the parameters vary, and the effect of the parameter variation to the system reliability is analyzed. Results show that (i) the comprehensive transmission error has the largest influence on system dynamic reliability, while the mass density has the least influence; (ii) the dynamic reliability of the gear transmission system reduces, and the dispersion degree increases, with the increase of the variation of the parameters; (iii) for the gear transmission system of the $1.5 \mathrm{MW}$ wind turbine, planetary gear system has the highest dynamic reliability, followed by the medium speed level gears, while high speed level gear is the least reliable. At the same time, in the planetary gear system, internal gears have the highest reliability, followed by the planetary gear, while the sun gear is the least reliable. In the medium and high speed level gears, large gears are more dynamic-reliable than the small ones.

\section{Conflict of Interests}

The authors declare that there is no conflict of interests regarding the publication of this paper.

\section{Acknowledgments}

This work was supported in part by the Major State Basic Research Development Program 973 (no. 2012CB215202), the National Natural Science Foundation of China (no. 51205046), and the Fundamental Research Funds for the
Central Universities. The constructive comments provided by the anonymous reviewers and the editors are also greatly appreciated.

\section{References}

[1] W. Musial, S. Butterfield, and B. McNiff, "Improving wind turbine gearbox reliability," in Proceedings of the European Wind Energy Conference, Milan, Italy, May 2007.

[2] L. Katafygiotis and S. H. Cheung, "Wedge simulation method for calculating the reliability of linear dynamical systems," Probabilistic Engineering Mechanics, vol. 19, no. 3, pp. 229-238, 2004.

[3] L. Katafygiotis and S. H. Cheung, "Domain decomposition method for calculating the failure probability of linear dynamic systems subjected to gaussian stochastic loads," Journal of Engineering Mechanics, vol. 132, no. 5, pp. 475-486, 2006.

[4] P. Liu and Q.-F. Yao, "Efficient estimation of dynamic reliability based on simple additive rules of probability," Engineering Mechanics, vol. 27, no. 4, pp. 1-4, 2010.

[5] H.-W. Qiao, Z.-Z. Lu, A.-R. Guan, and X.-H. Liu, "Dynamic reliability analysis of stochastic structures under stationary random excitation using hermite polynomials approximation," Engineering Mechanics, vol. 26, no. 2, pp. 60-64, 2009.

[6] A. Lupoi, P. Franchin, and M. Schotanus, "Seismic risk evaluation of RC bridge structures," Earthquake Engineering \& Structural Dynamics, vol. 32, no. 8, pp. 1275-1290, 2003.

[7] P. Franchin, "Reliability of uncertain inelastic structures under earthquake excitation," Journal of Engineering Mechanics, vol. 130, no. 2, pp. 180-191, 2004.

[8] J. L. M. Peeters, D. Vandepitte, and P. Sas, "Analysis of internal drive train dynamics in a wind turbine," Wind Energy, vol. 9, no. 1-2, pp. 141-161, 2006.

[9] Z. Caichao, H. Zehao, T. Qian, and T. Yonghu, "Analysis of nonlinear coupling dynamic characteristics of gearbox system about wind-driven generator," Chinese Journal of Mechanical Engineering, vol. 41, no. 8, pp. 203-207, 2005.

[10] D. T. Qin, Z. K. Xing, and J. H. Wang, "Optimization design of system parameters of the gear transmission of wind turbine based on dynamics and reliability," Chinese Journal of Mechanical Engineering, vol. 44, no. 7, pp. 24-31, 2008.

[11] D.-T. Qin, X.-G. Gu, J.-H. Wang, and J.-G. Liu, "Dynamic analysis and optimization of gear trains in a megawatt level wind turbine," Journal of Chongqing University, vol. 32, no. 4, pp. 408414, 2009.

[12] X.-L. Jiang and C.-F. Wang, "Stochastic volatility models based Bayesian method and their application," Systems Engineering, vol. 23, no. 10, pp. 22-28, 2005.

[13] H. T. Chen, X. L. Wu, D. T. Qin, J. Yang, and Z. Zhou, "Effects of gear manufacturing error on the dynamic characteristics of 
planetary gear transmission system of wind turbine," Applied Mechanics and Materials, vol. 86, pp. 518-522, 2011.

[14] H. Wang, B. Xing, and H. Luo, "Ranflow counting method and its application in fatigue life prediction," Mining \& Processing Equipment, vol. 34, no. 3, pp. 95-97, 2006.

[15] Z.-B. Wang, G.-F. Zhai, X.-Y. Huang, and D.-X. Yi, “Combination forecasting method for storage reliability parameters of aerospace relays based on grey-artificial neural networks," International Journal of Innovative Computing, Information and Control, vol. 9, no. 9, pp. 3807-3816, 2013.

[16] Y.-K. Lin, L. C.-L. Yeng, S.-I. Chang, and S.-R. Hsieh, "Network reliability evaluation for computer networks: a case of the Taiwan advanced research and education network," International Journal of Innovative Computing, Information and Control, vol. 9, no. 1, pp. 257-268, 2013.

[17] R. M. Ignatishchev, "Refinement of outlook at contact strength of gears and new potentiality of increase of their reliability," Problemy Mashinostroeniya i Nadezhnosti Mashin, no. 6, pp. 6870, 1992.

[18] J. N. Yang and M. D. Liu, "Residual strength degradation model and theory of periodic proof tests for graphite/epoxy laminates," Journal of Composite Materials, vol. 11, no. 2, pp. 176-203, 1977.

[19] W. Sun, H. Gao, and O. Kaynak, "Finite frequency $H_{\infty}$ control for vehicle active suspension systems," IEEE Transactions on Control Systems Technology, vol. 19, no. 2, pp. 416-422, 2011.

[20] W. Sun, Y. Zhao, J. Li, L. Zhang, and H. Gao, "Active suspension control with frequency band constraints and actuator input delay," IEEE Transactions on Industrial Electronics, vol. 59, no. 1, pp. 530-537, 2012. 


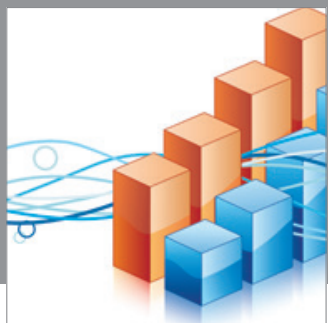

Advances in

Operations Research

mansans

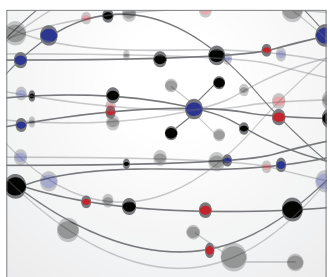

The Scientific World Journal
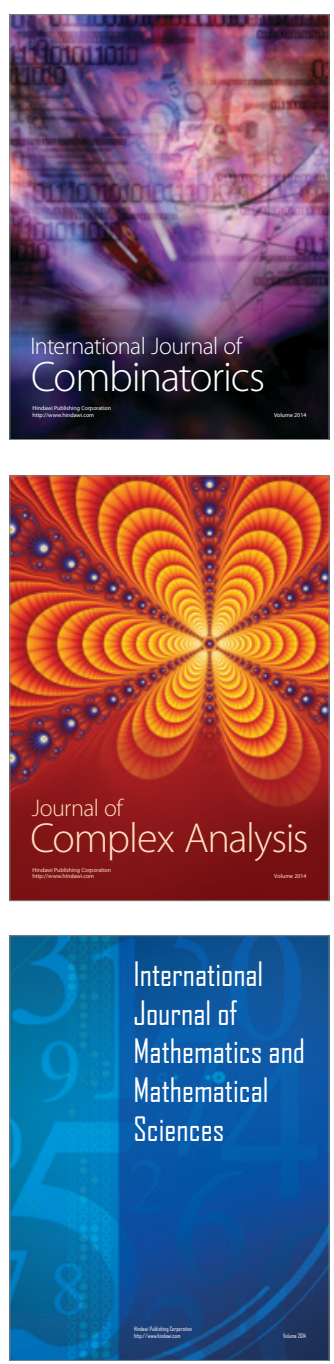
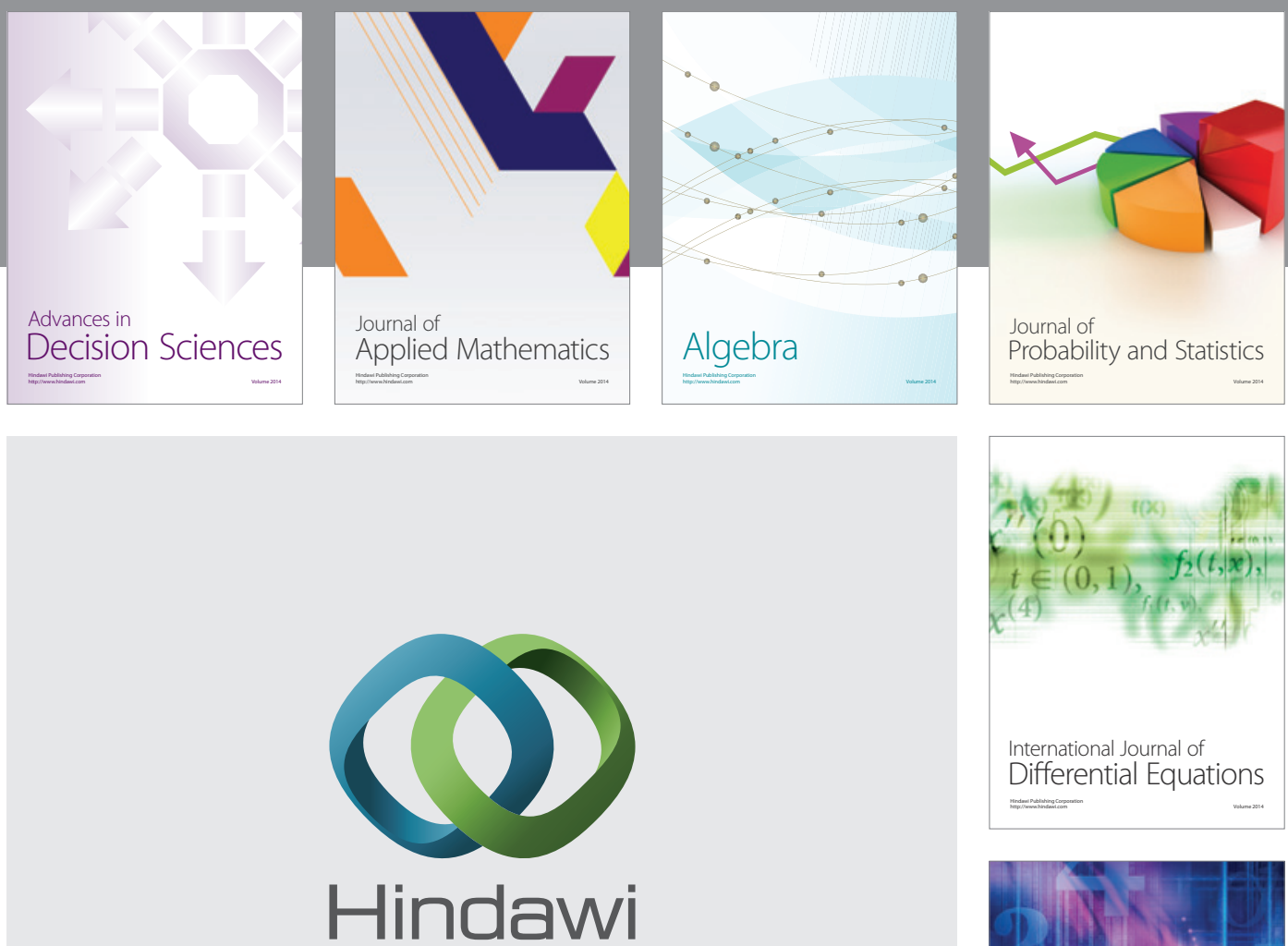

Submit your manuscripts at http://www.hindawi.com
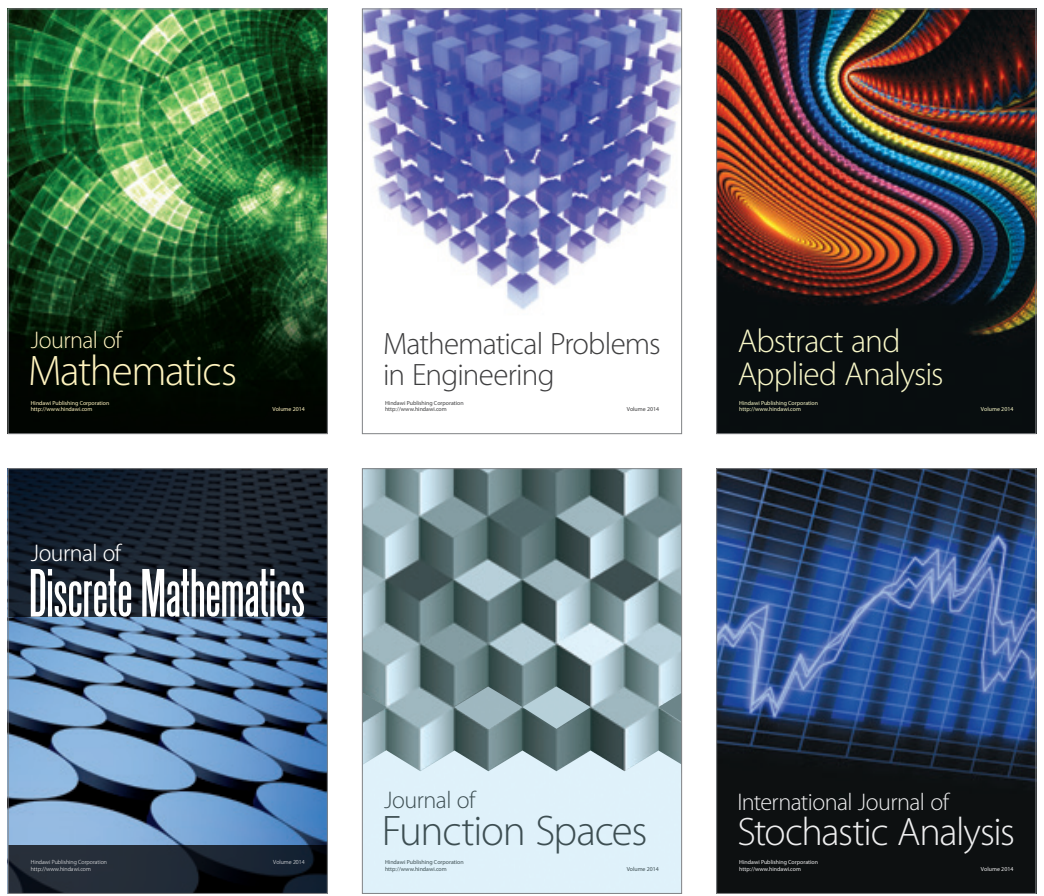

Journal of

Function Spaces

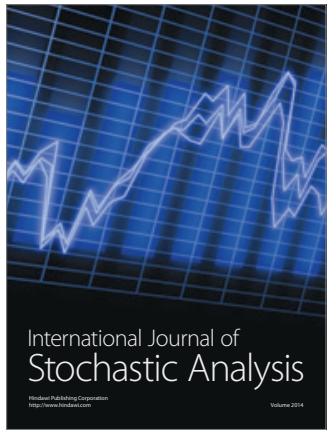

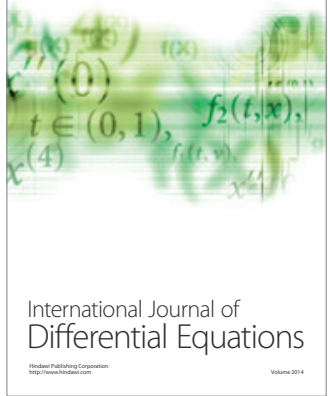
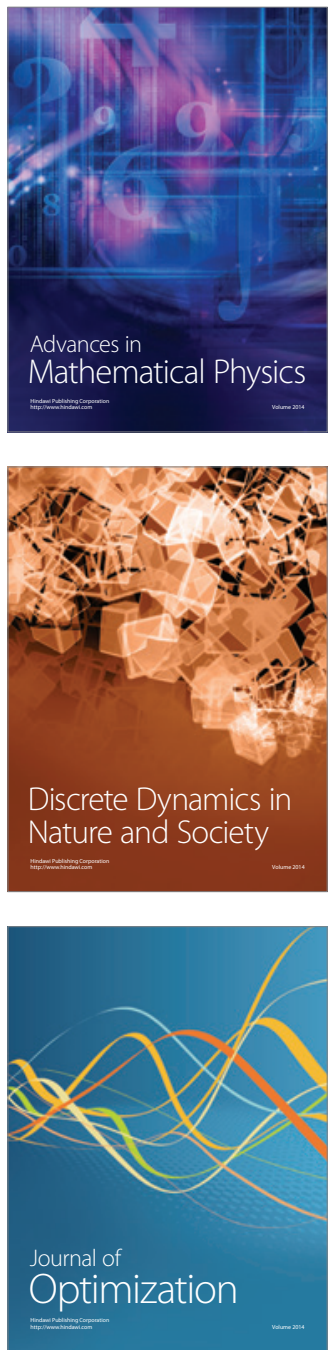